\title{
Effect of Mineral, Organic and Bio- Fertilization on Yield and Quality of Parsley (Petroselluinam sativam, L.)
}

\author{
"Radwan, F. I .," A. I. Abido," E. H. Shaben and Drakhshan I. saeed" \\ * plant production Dept., Fac. Agric. (Saba Basha) Alexandria University, Egypt \\ ${ }^{* *}$ Medicinal and Aromatic Res. Dept, A. R. C. Alexandria, Egypt, Div. of Medicinal and \\ Aromatic plants
}

\begin{abstract}
Two field experiments were carried out at the Experimental Farm of the Faculty of Agriculture (Saba Basha), Alexandria University, at Abees region, Alexandria. Egypt during the seasons of 2012/2013 and 2013/2014 to study the effect of NPK fertilizers, organic manure levels and biofertilizers on the growth parameters, chemical composition, and majors compounds of the essential oil of Parsley (Petroselinum sativum, L.). The applied experimental design was randomize complete blocks design with three replicates. The main results could be summarized as follows: (1) The fertilization treatments differently affected the mean values of all studied characters, (2) The application with organic manure (2 ton)/fed $+50 \%$ NPK + Cerealine + phoshorein and organic manure (4 ton)/fed $+50 \%$ NPK + Cerealine + phosphorein significantly increased plant height, leaf area index, fresh and dry weight at two cuts as well as chlorophyll a \& b, chemical composition (N, P and K\%) and Vitamin C content during both seasons. (3) However, the application organic manure 2 ton org/fed $+75 \%$ NPK + phosphorein gave the highest of major's compounds (apiol, myristien, B- pinene and B-phellandrene \%) during 2012/2013 season. This investigation suggests the need for more studies concerning the effect of NPK fertilizer organic manure and biofertilization on Parsley plant under different environments using different types of soil to reach the optimum combination to achive the best yield.
\end{abstract}

Key words: Mineral, Organic, biofertilization, Parsley, major's compounds oil.

\section{INTRODUCTION}

Petroslinum sativum Mill (parsley) Fam. Apiaceae is a widely cultivated herb used extensively for garnishing and seasoning foods, and for production of an essential oil. Fresh parsley is one of the most popular green herbs. The mature seed is steam distilled to produce parsley seed's, oil and parsley herb oil comes from the plant bearing immature seeds. Parsley herb oil has flavor more like the fresh leaves and is in greater demand than seed oil, which is often distilled from aged seed of low germinability (Simon et al., 1984). The root may, also, be harvested for use as a medicinal herb. Parsley combines well with most foods except sweets. It has a mild taste blends other flavors together, and has a high nutrient's content and used in medicinal, household cosmetic and fragrance (Rashed, 2002).

Fertilization is one of the most important factors limiting the productivity of plants. The intensive use of expensive mineral fertilizers in recent gears results in environmental pollution problems. However, Chemical fertilizers at extremely high rates for along period decreased the potential activity of microflora and the stability of soil organic matter (Hussien, 1995). Additionally, organic manures are in the form of compost, animals manure, farmyard manure (FYM) and green manure organic materials are generally added to soils to improve their physical and chemical properties. They enhance the soil fertility by their composition of macro and microelements, amino acid, organic acids, sugars and organic matter (Abou El- Fadl et al., 1968). Furthermore, biofertilization is an important factor being used to produce products without some mineral fertilizer that cause environmental pollution problems, and high 
rates of it leads to decrease the potential activity of microflora and the mobility of organic matters. Hence, the attention has been focused on the researches of bio- fertilization to safe alternative to specific chemical fertilizers. Biofertilizers play vital role for increasing the number of microorganisms and accelerate certain microbial process in the rhizosphere of inoculated soil of plants which can change the available forms of some nutrients tobe plants (Kandeel et al., 2001, Rashed, 2002; Mohamed and Abdu, 2004).

This research, however, is an attempt to find out the best fertilization treatments (chemical fertilizer, organic manure and biofertilizer) on the vegetative growth and chemical composition of psarley (Petroselinum sativum, Mill).

\section{MATERIALS AND METHODS}

Two filed experiments were carried out at the Experimental Farm of Faculty of Agric. (Saba Basha) Alexandria University, at Abees region, Alexandria, Egypt, during, the two growing seasons of 2012/2013 and $2013 / 2014$ to study the effect of fertilization treatments (chemical fertilizer, organic manure and bio- fertilization) on growth and chemical composition of parsley( Petroselinum sativum Mill) plants. The experimental design was a complete randomized block design with three replicates.

The parsley seeds were sown on November $11^{\text {th }}$ and $14^{\text {th }}$ in the two growing seasons, respectively. The plots area of each was 4 square meters $(2.0 \mathrm{~m} \times 2.0 \mathrm{~m})$ with 3 rows, the distance between the rows was $50 \mathrm{~cm}$ and $10 \mathrm{~cm}$ between plants.

The chemical fertilizers were applied as ammonium sulphate $(20.5 \% \mathrm{~N})$, calcium superphosphate $\left(15.5 \% \mathrm{P}_{2} \mathrm{O}_{5}\right)$ and potassium sulphate $\left(48 \% \mathrm{~K}_{2} \mathrm{O}\right)$ at the rates of $(100,100$ and $50 \mathrm{~kg} / \mathrm{fed}$, respectively) which are the recommended dose.

The used biofertilization of bacteria were phosphorein (Bacillusmegatheruim phosphorus dissolving bacteria P.D.B.), cerealine (Azospirillum Lipoferum and Azotobacter chroococcum) which supplied by National Research Center. The inoculation, with phosphorein and cerealine was preformed by coating parsley seed with each product individually using a sticking substance (Arbic gum at $5 \%$ ) just before sowing.

The organic fertilization (Sheap manure) was carried at the rates of 2 and 4 tons/fed, which were applied through the soil preparation before sowing.

The recommended dose of NPK was divided in two equal parts, the first one was applied one month after sowing and the second one was applied after the first cut.

The tested treatments were conducted as follows:

$\mathrm{F}_{1}: 100 \%$ NPK (control).

$F_{2}: 75 \% N P K+$ cerealine. 
$\mathrm{F}_{3}: 50 \% \mathrm{NPK}$ + cerealine + phosphorein.

$\mathrm{F}_{4}$ : Organic manure (2 ton/fed) $+75 \% \mathrm{NPK}$.

$\mathrm{F}_{5}$ : Organic manure $(2$ ton/fed $)+75 \% \mathrm{NPK}+$ cerealine.

$\mathrm{F}_{6}$ : Organic manure $(2$ ton/fed $)+75 \% \mathrm{NPK}+$ phosphorein .

$\mathrm{F}_{7}$ : Organic manure $(2$ ton/fed $)+50 \% \mathrm{NPK}+$ cerealine+ phosphorein

$\mathrm{F}_{8}$ : Organic manure (4 ton/fed) $+75 \% \mathrm{NPK}$.

$F_{9}$ : Organic manure (4 ton/fed) $+75 \%$ NPK + cerealine.

$\mathrm{F}_{10}$ : Organic manure (4 ton/fed) $+75 \% \mathrm{NPK}+$ phosphorein.

$F_{11}$ : Organic manure $(4$ ton/fed $)+50 \%$ NPK + cerealine+ phosphorein.

The physical and chemical characteristics of the experimental soil and used sheep manure composition are given in Tables (1) and (2). The soil was ananlyzed according to be methods deseribed by Page et al. (1982)

Table (1): The physical and chemical properties of the experimental soil in 2012/2013 and 2013/2014 seasons

\begin{tabular}{|c|c|c|}
\hline \multirow{2}{*}{ Soil properties } & \multicolumn{2}{|c|}{ Values } \\
\hline & $2012 / 2013$ & 2013/2014 \\
\hline \multicolumn{3}{|l|}{ A- Particle size distribution (\%) } \\
\hline Sand & 15.00 & 14.80 \\
\hline Silt & 42.00 & 42.20 \\
\hline Clay & 43.00 & 43.00 \\
\hline Soil texture & Clay loam & Clay loam \\
\hline \multicolumn{3}{|l|}{ B- Chemical properties } \\
\hline$\overline{\mathrm{pH}(1: 1)}$ & 7.90 & 7.80 \\
\hline EC (1:1) dS/m & 2.20 & 2.10 \\
\hline 1- Soluble cations (1:2) (Cmol/kg soil) & & \\
\hline $\mathrm{K}^{+}$ & 0.90 & 0.95 \\
\hline $\mathrm{Ca}^{++}$ & 4.15 & 4.20 \\
\hline $\mathrm{Mg}^{++}$ & 3.10 & 3.15 \\
\hline $\mathrm{Na}^{++}$ & 8.10 & 8.20 \\
\hline \multicolumn{3}{|l|}{ 2- Soluble anions (1:2) (Cmol/kg soil) } \\
\hline $\mathrm{CO}_{3}^{-}+\mathrm{HCO}_{3}^{-}$ & 2.70 & 2.60 \\
\hline $\mathrm{CL}^{-}$ & 11.50 & 11.70 \\
\hline $\mathrm{SO}_{4}^{-}$ & 0.50 & 0.48 \\
\hline Calcium carbonate, $\%$ & 7.70 & 7.80 \\
\hline Organic matter, \% & 1.00 & 0.90 \\
\hline Total nitrogen, \% & 0.45 & 0.47 \\
\hline Avaliable Phosphorus (mg/kg) & 3.70 & 3.80 \\
\hline Avaliable K (mg/kg) & 162.3 & 170.1 \\
\hline
\end{tabular}


Also, the chemical analysis of the organic manure was carried out according the method of Jackson (1967).

Table (2): Analysis of the applied organic manure (sheep manure)

\begin{tabular}{cc}
\hline $\mathrm{pH}$ & 7.5 \\
\hline O.M (\%) & 23.20 \\
\hline O.C (\%) & 21.00 \\
\hline Total (N\%) & 2.20 \\
\hline Total (P\%) & 1.15 \\
\hline Total (K\%) & 1.45 \\
\hline C/N ratio & $11.5: 1$ \\
\hline
\end{tabular}

At harvest dates on January $7^{\text {th }}$ and February $6^{\text {th }}$ in the two season, guarded plants were randomly taken from each plots and the following characteristics were recorded:

1. Plant height $(\mathrm{cm})$.

2. Fresh and dry weights of aerial parts/plant $(\mathrm{g})$.

3. Leaf area index $\left(\mathrm{cm}^{2}\right)$.

4. Chlorophyll ( $a$ and $b), \mathrm{mg} / \mathrm{g}$ fresh weight were determined in fresh leaves samples of the fifth leaf from top at harvest and after 30 days for parsley, using the method by Moran (1982).

5. The $\mathrm{N}, \mathrm{P}$ and $\mathrm{K}$ contents were determined in the acid digested solution which was prepared according to Hach et al. (1985) using a mixture of hydrogen peroxide and sulfuric acid (4:10).

- Elements extraction was made on a known weight of the dried samples (0.2 mg).

- Nitrogen was determined using the microkjeladhl method according to Black (1983).

- Phosphorus was determined colorimetrically using the method described by Jackson (1967) and Potassium was estimated using flame photometer method according to Richards (1954).

1. Vitamin (C) content was determined in filtered juice samples and expressed as $(\mathrm{mg})$ ascorbic acid/100 $\mathrm{ml}$ fresh juice as described by (A.O.A.C., 1965).

2. The percentage of major constituents (Apiole, myristiein, B-pinene, and B- phellandrene) were estimated by measuring the peak area of the different compounds of the chromatogram according to Heftman (1967) and Gunther and Joseph (1978).

The obtained data were, statistically, analyzed for ANOVA, and L.S.D. values were calculated to test the differences between the studied treatments according to Gomez and Gomez (1984).

\section{RESULTS AND DISCUSSION}

\section{A- Growth parameters and yield:}

The obtained results, given in Tables ( 3 and 4) cleared, that fertilizer treatments exhibited a significant effect on all estimated traits in both seasons. 
Application of $\left(\mathrm{F}_{6}\right)$ treatment organic manure $(2$ ton)/fed $+50 \% \mathrm{NPK}+$ cerealine + phosphorein and $\left(F_{11}\right)$ organic manure $(4$ ton $) / f e d+50 \%$ NPK + cerealine + phosphorin significantly, increased plant height, leaf area index, fresh and (g), dry weight $(\mathrm{g})$ at two cuts as well chlorophyll $\mathrm{a}, \mathrm{b}$ in both seasons. It could be concluded that this positive effect on growth characters and chlorophyll $a, b$ in response to sheep manure levels, may be attributed to increasing maentration in plant tissues (Opera and Asigebu 1996). Also, the phosphate solubilizing bacteria (phasphorein) and nitrogen fixing (cerealine) may increase the synthesis of endogenous phytothormones i.e. IAA, GAs and CKs which play an important role in formation of a big active root system which allow more nutrients, uptake. The previous results agree, more or less, with the findings of Rashed (2002) on parsley, Gad (2001) on Anelthum graveolens; Mohammad et al. (2012) on pimpinella anisum; Abdel- Latif. (2002) on Caruim carvi and Kandeel et al. (2001) and Mohamed and Abdu (2004) on Foeniculum vulgare.

\section{B- Chemical composition and vitamin (C):}

The data in Table (4) showed that all treatments of fertilization, affected chemical composition (N, P and K\%) and vitamin, (C) content in both seasons. It is clear from data that the highest mean values of chemical composition $(\mathrm{N}, \mathrm{P}$ and $\mathrm{K} \%)$ and vitamin $(\mathrm{C})$ content, resulted from the treatments of $\left(\mathrm{F}_{7}\right) 2$ ton organic manure/fed $+50 \% \mathrm{NPK}+$ cerealine + phosphorein and $\left(F_{11}\right) 4$ ton/fed organic manure $+50 \% \mathrm{NPK}+$ cerealine+ phosphorein in both seasons.

The increment of chemical composition ( $\mathrm{N}, \mathrm{P}$ and $\mathrm{K} \%$ ) and vitamin (C) content of plant's leaves using the treatments of organic manure and half dose of NPK and biofertilization; may be attributed to increase in the occupancy root zone of plant as a results of adding fertilization treatments which reflected on nutrients uptake by plants and confirm the previous of vegetative growth. Similar results, more or less were obtained by Kandeel et al. (2001) and Abou El- Maged et al. (2008) on fennel; Rashed (2002) on Petroselinium sativum, Likwise the results showed significant differences for organic manure + biofertilization in the both seasons, which gave the greatest values for all chemical composition.

\section{C- Major components percentage of essential oil:}

The effect of fertilization treatments on essential oil majors compounds (Apiol, Myristien, B. Pinene and B- Phellandrene) percentages are shown in Table (5). The results indicated that using fertilization treatments had significant effect on the studied majors compounds percentage of parsley oil. The application of 2 tons organic manure fed $+75 \% \mathrm{NPK}+$ phosphorein; gave the highest percentage of majors compounds in 2012/2013 season. Similar results were reported by Darzi et al (2011) on anisum and Ismail et al. (2009) on majoram plant. 
Table (3) : Effect of fertilization treatments on vegetative growth at two cut during 2012/2013 and 2013/2014 seasons

\begin{tabular}{|c|c|c|c|c|c|c|c|c|c|c|c|c|}
\hline \multirow{3}{*}{ Treatments } & \multicolumn{4}{|c|}{ Plant height $(\mathrm{cm})$} & \multicolumn{4}{|c|}{ Leaf area index $\left(\mathrm{cm}^{2}\right)$} & \multicolumn{4}{|c|}{ Fresh weight (g) } \\
\hline & \multicolumn{2}{|c|}{$2012 / 2013$} & \multicolumn{2}{|c|}{$2013 / 2014$} & \multicolumn{2}{|c|}{$2012 / 2013$} & \multicolumn{2}{|c|}{$2013 / 2014$} & \multicolumn{2}{|c|}{$2012 / 2013$} & \multicolumn{2}{|c|}{$2013 / 2014$} \\
\hline & $1^{\text {st }}$ cut & $2^{\text {nd }}$ cut & $1^{\text {st }}$ cut & $2^{\text {nd }}$ cut & $1^{\text {st }}$ cut & $2^{\text {nd }}$ cut & $1^{\text {st }}$ cut & $2^{\text {nd }}$ cut & $1^{\text {st }}$ cut & $2^{\text {nd }}$ cut & $1^{\text {st }}$ cut & $2^{\text {nd }}$ cut \\
\hline$F_{1:}$ NPK (control) & $27.23 \mathrm{c}$ & $27.67 d$ & $25.00 d$ & $27.23 e d$ & $5.20 c$ & $3.71 \mathrm{c}$ & $4.90 \mathrm{~b}$ & $2.98 \mathrm{c}$ & $138.33 i$ & $124.00 d$ & $130 \mathrm{e}$ & $140 c$ \\
\hline$F_{2}: 75 \%$ NPK + Cerealine & $24.23 \mathrm{~g}$ & $25.17 \mathrm{~g}$ & $24.67 d$ & $24.53 \mathrm{~g}$ & $5.40 c$ & $3.90 \mathrm{~b}$ & $4.95 b$ & $3.38 b$ & $163.33 \mathrm{e}$ & $150.00 \mathrm{~b}$ & $190.5 b$ & $150 \mathrm{~b}$ \\
\hline $\mathbf{F}_{3}: 50 \% \mathrm{NPK}+$ Cerealine+ phosphorein. & $27.17 \mathrm{c}$ & $25.17 \mathrm{~g}$ & $24.67 d$ & $27.17 d$ & $5.81 \mathrm{~b}$ & $4.40 \mathrm{~b}$ & $5.10 \mathrm{~b}$ & $3.50 \mathrm{~b}$ & $198.33 d$ & $130.00 \mathrm{c}$ & $170 \mathrm{c}$ & $153 \mathrm{~b}$ \\
\hline F4: organic manure (2ton/fed) $+75 \%$ NPK & $27.47 \mathrm{~b}$ & $26.17 f$ & $24.33 \mathrm{e}$ & $27.73 \mathrm{c}$ & $4.67 d$ & $3.10 d$ & $4.70 \mathrm{c}$ & $3.17 \mathrm{~b}$ & $125.00 \mathrm{~b}$ & $110.00 \mathrm{e}$ & 160cd & $146 \mathrm{c}$ \\
\hline$F_{5}:$ organic manure $(2$ ton $/ \mathrm{fed})+75 \% \mathrm{NPK}+$ cerealine & $27.64 \mathrm{~b}$ & $28.17 \mathrm{c}$ & $27.83 b$ & $26.77 \mathrm{e}$ & $4.70 \mathrm{~d}$ & $3.15 d$ & $4.55 \mathrm{c}$ & $3.20 \mathrm{~b}$ & $216.67 \mathrm{cb}$ & $140.00 \mathrm{~b}$ & $190 \mathrm{c}$ & $154 \mathrm{~b}$ \\
\hline $\mathrm{F}_{6}:$ organic anure(2ton/fed)+75\%NPK+ phosphorein & $26.37 d$ & $28.83 b$ & $27.33 b$ & $27.37 \mathrm{~cd}$ & $5.21 \mathrm{~b}$ & $4.30 \mathrm{~b}$ & $5.15 b$ & $3.45 b$ & $211.67 \mathrm{c}$ & $150.00 \mathrm{~b}$ & $180 \mathrm{c}$ & $144 \mathrm{c}$ \\
\hline$F_{7}:$ organic anure $(2$ ton $/$ fed $)+50 \% N P K+$ Cerealine+ phosphorein & $28.77 a$ & $2967 a$ & $29.44 a$ & $28.97 a$ & $5.20 \mathrm{a}$ & $5.00 \mathrm{a}$ & $5.50 \mathrm{a}$ & $4.10 \mathrm{a}$ & $231.67 a$ & $161.16 \mathrm{a}$ & $215 a$ & $165 a$ \\
\hline$F_{8}:$ organic manure $(4$ ton/fed $)+75 \% N P K$ & $25.73 f$ & $26.33 f$ & $25.83 \mathrm{c}$ & $25.73 f$ & $5.75 b$ & $4.15 b$ & $5.11 \mathrm{~b}$ & $3.36 \mathrm{~b}$ & $156.67 \mathrm{~g}$ & 115.00de & $155 d$ & $144 \mathrm{c}$ \\
\hline$F_{9}:$ Org.(4ton/fed)+75\%NPK+ Cerealine & $26.07 e$ & $26.83 e$ & $25.33 \mathrm{c}$ & $26.07 f$ & $5.80 \mathrm{~b}$ & $4.20 \mathrm{~b}$ & $5.16 \mathrm{~b}$ & $3.26 \mathrm{~b}$ & $185.00 \mathrm{~d}$ & $135 . c$ & $195 \mathrm{~b}$ & $155 \mathrm{~b}$ \\
\hline$F_{10}:$ organic manur(4ton/fed)+75\%NPK+ phosphorein & $23.30 \mathrm{~h}$ & $28.83 b$ & $24.67 d$ & $24.30 \mathrm{~g}$ & $5.90 \mathrm{~b}$ & $4.18 \mathrm{~b}$ & $5.10 \mathrm{~b}$ & $3.30 \mathrm{~b}$ & 195.33d & $137.00 \mathrm{c}$ & $155 d$ & $148 \mathrm{~b}$ \\
\hline$F_{11}:$ organic manur(4ton/fed)+50\%NPK+ Cerealine+phosphorein & $28.90 a$ & $35.00 \mathrm{a}$ & $30.00 \mathrm{a}$ & $29.63 a$ & $6.00 a$ & $5.01 \mathrm{a}$ & $5.51 \mathrm{a}$ & $4.11 \mathrm{a}$ & $225.33 a$ & $156.00 \mathrm{a}$ & $170 \mathrm{c}$ & $165 a$ \\
\hline L.S.D. (0.05) & 0.25 & 0.30 & 0.40 & 0.45 & 0.35 & 0.40 & 0.30 & 0.40 & 10.30 & 9.10 & 9.30 & 8.90 \\
\hline
\end{tabular}

${ }^{*}$ Means followed by the same letter (s) in each column are not significantly different at 0.05 level of probability.

Table (3) : Cont'd.

\begin{tabular}{|c|c|c|c|c|c|c|c|c|}
\hline \multirow{3}{*}{ Treatments } & \multicolumn{4}{|c|}{ Dry weight (g) } & \multicolumn{4}{|c|}{ Chlorophyll (mg/g) } \\
\hline & \multicolumn{2}{|c|}{$2012 / 2013$} & \multicolumn{2}{|c|}{$2013 / 2014$} & \multicolumn{2}{|c|}{ 2012/2013 } & \multicolumn{2}{|c|}{ 2013/2014 } \\
\hline & $1^{\text {st }}$ cut & $2^{\text {nd }}$ cut & $1^{\text {st }}$ cut & $2^{\text {nd }}$ cut & $a$ & b & $a$ & b \\
\hline$F_{1:}$ NPK + control & $45.77 \mathrm{e}$ & $38.38 \mathrm{~g}$ & $30.40 f$ & $34.70 \mathrm{~h}$ & $1.60 \mathrm{~d}$ & $0.39 d$ & $1.58 \mathrm{~d}$ & $0.48 \mathrm{~b}$ \\
\hline$F_{2}: 75 \%$ NPK + Cerealine & $46.53 d$ & $41.20 \mathrm{c}$ & $55.60 \mathrm{~b}$ & $44.15 \mathrm{~b}$ & $1.62 \mathrm{c}$ & $0.31 \mathrm{e}$ & $1.51 \mathrm{f}$ & $0.33 d$ \\
\hline$F_{3}: 50 \% N P K+C e r e a l i n e+$ phosphorein. & $42.07 \mathrm{~g}$ & $39.80 \mathrm{~d}$ & $50.70 \mathrm{c}$ & $35.90 \mathrm{~g}$ & $1.65 c$ & $0.39 d$ & $1.55 \mathrm{e}$ & $0.36 \mathrm{c}$ \\
\hline F4: organic manure (2ton/fed) + 75\% NPK & $55.83 \mathrm{~b}$ & $40.15 d$ & $51.8 \mathrm{c}$ & $52.60 \mathrm{c}$ & $1.70 \mathrm{bc}$ & $0.40 d$ & $1.60 d$ & $0.39 \mathrm{c}$ \\
\hline$F_{5}:$ organic manure (2ton/fed) $+75 \%$ NPK+ Cerealine & $52.80 \mathrm{~b}$ & $41.20 \mathrm{c}$ & $35.70 \mathrm{~g}$ & $36.70 f$ & $1.75 \mathrm{bc}$ & $0.50 \mathrm{~b}$ & $1.58 \mathrm{~d}$ & $0.48 \mathrm{~b}$ \\
\hline$F_{6}:$ organic manure (2ton/fed)+75\%NPK+ phosphorein & $56.33 b$ & $40.60 \mathrm{c}$ & $40.70 \mathrm{e}$ & $38.90 \mathrm{e}$ & $1.74 \mathrm{bc}$ & $0.52 b$ & $1.55 \mathrm{e}$ & $0.49 \mathrm{~b}$ \\
\hline$F_{7}:$ organic manure (2ton/fed) $+50 \% N P K+$ Cerealine+ phosphorein & $62.80 \mathrm{a}$ & $53.38 \mathrm{a}$ & $71.20 \mathrm{a}$ & $54.41 \mathrm{a}$ & $2.18 \mathrm{a}$ & $0.60 \mathrm{a}$ & $1.90 \mathrm{a}$ & $0.62 \mathrm{a}$ \\
\hline$F_{8}:$ organic manure (4ton/fed)+75\%NPK & $42.43 \mathrm{~g}$ & $36.30 f$ & $42.60 d$ & $40.60 d$ & $1.74 \mathrm{bc}$ & $0.42 d$ & $1.66 c$ & $0.52 \mathrm{~b}$ \\
\hline$F_{9}:$ Org.(4ton/fed)+75\%NPK+ Cerealine & $56.73 b$ & $41.70 \mathrm{c}$ & $35.40 \mathrm{~g}$ & $36.40 f$ & $1.78 b$ & $0.46 c$ & $1.72 b$ & $0.48 \mathrm{~b}$ \\
\hline$F_{10}:$ organic manure (4ton/fed)+75\%NPK+ phosphorein & $49.47 \mathrm{c}$ & 37.40 & $34.70 \mathrm{~g}$ & $33.90 \mathrm{i}$ & $1.72 \mathrm{bc}$ & $0.40 d$ & $1.68 c$ & $0.50 \mathrm{~b}$ \\
\hline$F_{11}:$ organic manure (4ton/fed)+50\%NPK+ cerealine+phosphorein & $62.07 a$ & $54.20 \mathrm{a}$ & $71.70 \mathrm{a}$ & $54.15 a$ & $2.20 \mathrm{a}$ & $0.16 a$ & $1.92 \mathrm{a}$ & $0.63 \mathrm{a}$ \\
\hline L.S.D. (0.05) & 1.20 & 1.00 & 1.20 & 1.00 & 0.10 & 0.03 & 0.03 & 0.03 \\
\hline
\end{tabular}

${ }^{*}$ Means followed by the same letter (s) in each column are not significantly different at 0.05 level of probability. 
Table (4) : Chemical composition (N, P and K percentages) and vitamin (C) as influenced by fertilization treatments during 2013 and 2014 seasons

\begin{tabular}{|c|c|c|c|c|c|c|c|c|}
\hline \multirow[t]{2}{*}{ Treatments } & \multicolumn{2}{|c|}{$\mathrm{N} \%$} & \multicolumn{2}{|c|}{$\mathbf{P} \%$} & \multicolumn{2}{|c|}{$\mathbf{K} \%$} & \multicolumn{2}{|c|}{$\begin{array}{c}\text { Vitamin (C) } \\
\text { Mg/100ml fresh Juice }\end{array}$} \\
\hline & 2013 & 2014 & 2013 & 2014 & 2013 & 2014 & 2013 & 2014 \\
\hline$F_{1:}$ NPK ( control ) & $2.60 \mathrm{~d}$ & $2.75 d$ & $0.590 . \mathrm{gh}$ & 0.602 ef & $2.37 e$ & $2.45 \mathrm{e}$ & $107.08 p$ & $109.30 f$ \\
\hline$F_{2}: 75 \%$ NPK + Cerealine & $2.75 \mathrm{~cd}$ & $3.10 \mathrm{c}$ & $0.600 \mathrm{eg}$ & $0.617 d$ & $2.58 d$ & $2.50 e$ & $109.10 \mathrm{eb}$ & $110.20 \mathrm{ef}$ \\
\hline$F_{3}: 50 \% N P K+C e r e a l i n e+$ phosphorein. & $2.77 \mathrm{~cd}$ & $3.20 \mathrm{c}$ & $0.595 f$ & $0.605 \mathrm{e}$ & $2.60 d$ & $2.65 d$ & $109.50 \mathrm{e}$ & 110.70def \\
\hline F4: organic manure (2ton/fed) $+75 \%$ NPK & $2.56 \mathrm{e}$ & $2.90 d$ & $0.585 h$ & $0.595 f$ & $2.67 d$ & $2.70 d$ & 110.30de & $111.40 \mathrm{cde}$ \\
\hline$F_{5}:$ organic manure (2ton/fed)+75\% NPK+ Cerealine & $2.80 \mathrm{bcd}$ & $2.30 \mathrm{c}$ & $0.610 \mathrm{de}$ & $0.622 \mathrm{~d}$ & $2.81 \mathrm{C}$ & $2.85 \mathrm{c}$ & 111.70de & 112.20cde \\
\hline$F_{6}:$ organic manure (2ton/fed)+75\%NPK+ phosphorein & $3.10 \mathrm{~b}$ & $3.60 \mathrm{~b}$ & $0.635 b$ & $0.650 \mathrm{~b}$ & $2.87 \mathrm{bc}$ & $2.90 \mathrm{c}$ & $112.30 d$ & $112.80 \mathrm{~cd}$ \\
\hline$F_{8}:$ organic manure (4ton/fed)+75\%NPK & $2.85 \mathrm{bc}$ & $3.50 b$ & $0.620 \mathrm{~cd}$ & $0.635 \mathrm{c}$ & $2.67 d$ & $2.70 d$ & $112.90 \mathrm{~d}$ & $113.40 \mathrm{c}$ \\
\hline$F_{9}:$ Org.(4ton/fed)+75\%NPK+ Cerealine & $2.90 \mathrm{bc}$ & $3.50 b$ & $0.590 \mathrm{gh}$ & $0.604 \mathrm{ef}$ & $2.96 b$ & $3.20 \mathrm{~b}$ & $117.90 \mathrm{c}$ & $120.50 \mathrm{~b}$ \\
\hline$F_{10}:$ organic manure (4ton/fed)+75\%NPK+ hosphorein & $3.00 \mathrm{~b}$ & $3.70 \mathrm{~b}$ & $0.630 \mathrm{bc}$ & $0.650 \mathrm{~b}$ & $2.97 \mathrm{~b}$ & $3.20 \mathrm{~b}$ & $120.30 \mathrm{~b}$ & $122.40 \mathrm{~b}$ \\
\hline$F_{11}:$ organic manure $(4$ ton $/ \mathrm{fed})+50 \% \mathrm{NPK}+$ Cerealine+phosphorein & $3.90 \mathrm{a}$ & $3.30 \mathrm{a}$ & $0.670 \mathrm{a}$ & $0.680 \mathrm{a}$ & $3.40 \mathrm{a}$ & $3.55 a$ & $132.70 \mathrm{a}$ & $136.30 \mathrm{a}$ \\
\hline L.S.D. (0.05) & 0.20 & 0.25 & 0.012 & 0.010 & 0.015 & 0.012 & 2.40 & 2.30 \\
\hline
\end{tabular}

${ }^{\star}$ Means followed by the same letter (s) in each column are not significantly different at 0.05 level of probability.

Table ( 5): Effect of fertilization treatments on major components (\%) in Parsely oil of second season (2013/2014)

\begin{tabular}{|c|c|c|c|c|}
\hline Treatments & $\begin{array}{c}\text { Apiol } \\
(\%)\end{array}$ & $\begin{array}{c}\text { Myristicin } \\
(\%)\end{array}$ & $\begin{array}{c}\text { B- Pinene } \\
(\%)\end{array}$ & $\begin{array}{c}\text { B- Phellandrene } \\
(\%)\end{array}$ \\
\hline$F_{1:}$ NPK + control & $17.2 \mathrm{e}$ & $31.5 \mathrm{~d}$ & $12.5 \mathrm{~g}$ & $5.1 \mathrm{cb}$ \\
\hline$F_{2}: 75 \%$ NPK + Cerealine & $17.5 \mathrm{de}$ & $32.4 \mathrm{C}$ & $12.3 \mathrm{~g}$ & $5.3 b$ \\
\hline$F_{3}: 50 \% N P K+$ Cerealine+ phosphorein. & 17.4de & $33.2 \mathrm{c}$ & $14.1 \mathrm{c}$ & $5.3 b$ \\
\hline F4: organic manure (2ton/fed) $+75 \%$ NPK & $17.9 \mathrm{c}$ & $33.4 \mathrm{c}$ & $14.2 \mathrm{c}$ & $5.8 \mathrm{e}$ \\
\hline$F_{5}:$ organic manure (2ton/fed)+75\% NPK+ Cerealine & $18.3 b$ & $34.2 b$ & $14.8 \mathrm{~b}$ & $5.9 a$ \\
\hline$F_{6}:$ organic manure (2ton/fed)+75\%NPK+ phosphorein & $18.8 \mathrm{a}$ & $35.3 a$ & $15.1 \mathrm{a}$ & $6.00 \mathrm{a}$ \\
\hline$F_{7}:$ organic manure (2ton/fed)+50\%NPK+ Cerealine+ phosphorein & $18.4 \mathrm{~b}$ & $35.3 a$ & $13.7 d$ & $5.9 \mathrm{a}$ \\
\hline$F_{8}:$ organic manure $(4$ ton $/ \mathrm{fed})+75 \% \mathrm{NPK}$ & $17.9 \mathrm{c}$ & $34.2 b$ & $12.8 f$ & $5.2 b$ \\
\hline F9:Org.(4ton/fed)+75\%NPK+ Cerealine & $17.2 \mathrm{e}$ & $34.3 b$ & $12.7 f$ & $5.4 \mathrm{~b}$ \\
\hline$F_{10}:$ organic manure $(4 t o n / f e d)+75 \% N P K+$ phosphorein & $17.3 e$ & $33.5 \mathrm{c}$ & $12.7 f$ & $5.1 \mathrm{cb}$ \\
\hline$F_{11}:$ organic manure (4ton/fed)+50\%NPK+ Cerealine+phosphorein & 17.3 & $33.6 \mathrm{c}$ & $13.2 \mathrm{e}$ & $5.1 \mathrm{cb}$ \\
\hline L.S.D. (0.05) & 0.35 & 0.42 & 0.30 & 0.25 \\
\hline
\end{tabular}

* Means followed by the same letter (s) in each column are not significantly different at 0.05 level of probability. 


\section{REFERENCES}

Abdel- Latif, T. A. 2002. Effect of organic manure and biofertilizer on caraway plants (Carum carvi, L.) j. Agric. Sci. Mansoura Univ., 27 (5): 34593468.

Abou- El- Fadl, M., S. G. Rizk, A. F. Abdel Ghani, M. K. El- Mofty, M. F. A. Kader S. M. Shehata and F. A. Fdrag. 1968. Utilization of water gycointh as an organic manure with special refute water- bohne helminth. Microbical ARF, 3 (1): 27-34.

Abou- El- Magid, M. M., M. F. Zaki and S. D. Abou- Hussein 2008. Effect of organic manure and different lebels of saline irrigation water on growth, green yield and chemical content of sweet fennel. Aust. J. Basic\& Appl. Sci., 2 (1): 90- 98.

A.O. A. C. (Assocition of official Agricultural Chemists). 1965. Official and tentative Methods of analysis $10^{\text {th }}$ ed. 1008P. Washinton D. C.USA.

Black, C. A. 1983. Methods of soil Analysis Part 1and 32. Soil Sci. Soc. Amer Inc. Publ. Madison, Wisconsin, USA.

Darzi, M. T., M. R. Haj Seyed Hadi and F. Rejali 2011. Effect of vermicompost and phosphate bio- fertilizer application on yield and yield components in anise (Pimpinella anisum, L.) Iran. J. Med. Aroma plants, 4(50): 451465.

Gad, Wessam, M.A.M. 2001. Physiological studies on Foeniculum vulgare, Mill, and Anethum graveolens, L. M. Sc. Thesis, Fac. Agric Kaf- El- Sheikh, Tanta Univ. Egypt.

Gomez, K. A. and A. A. Gomez 1984. Statistical Procedures for Agricultural Research $2^{\text {nd }}$ edition . John Wiley \& Sons. Inc., New York.

Gunther, Z. and S. Josaph 1978. Hand Book Series in Chromatography CRC press. Inc.

Hach, C. C., S. V. Brayton and A. B. Kapelove 1985. Powerful kejeldahl nitrogen method using proxy mono sulfuric acid. J. Agric., Food Chem., 33: 1117-1123.

Heftman, E. 1967. Chromatography Reinhold Pub. Crop. New York.

Hussein, M. S. 1995. Response of coriander and dill to different nitrogen sources Egypt. J. Hort., 22 (1):1-10.

Ismail, A. G., E. M. Desouky, Y. Gamal, M. Galal, A. A. Arafa and A. M. Abou Seer 2009. Effect of biofertilizers and organic phosphorus amendment on growth and essential oil of marjoram (Mojorana hortensis, L.) Egypt. Acad, J. Biolog. Sci., 1 (1): 29- 38.

Jackson, M. L. (1967). Soil chemical analysis Prentice Hall of India, Private Limited New Delhi, p:115.

Kandeel, Y.R., Nofal, E.S., Menesi, F.A., Reda, K.A., Taher, M. and Zaki, Z.Y. 2001. Effect of some cultural practices on growth and chemical composition of Foeniculum vulgare, Mill. Proceeding $5^{\text {th }}$ Arabi. Horti. Conf. Ismalia, Egypt, March 24- 28 pp: 61-72.

Mohamed, M.A.H. and M. Abdu. 2004. Growth and oil production of fennel (Foeniculum vulgare Mill.), effect of irrigation and organic fertilization. Bio. Agric. and Hort., 22: 31-39.

Mohammad, T. D., H. S. M. Reza and F. Rejale 2012. Effect of the application of vermicompost and phosphate solubilizing bacterium on the 
morphological trait and seed yield of anise (Pimpinella anisum, L.) J. Medi. Plant Res., 6 (2): 215- 219.

Moran, R. 1982. Formula determination of chlorophyllous pigment extracted with N, Ndimethyl farmamide, Plant Physicl., 69:1376- 1381.

Opera, C. N. and J. E. Asigbu 1996. Nutrient content of poultry manures and the optimum role for Egyptian fruit yield in a weathered tropical Ultisol Bid., Agric.\& Hort., 13: 341- 350.

Rashed, Nahed, M. M. 2002. Effect of fertilization on the growth and storability of some aromatic plants. M. Sc. Thesis, Fac. Agric. Kafer EL-Sheikh, Tanta Univ, Egypt.

Richards, L. A. 1954. Diagnosis and improvement of saline and Alkaline soils. U.S.D.A. Agric. Hand Book No. 60. Gov. Print off.

Simon, J. E., A. F. Chadwick and L. E. Craker 1984. Herbs: An Indexed Bibliography. 1971-1980. The Scientific Literature on Selected Herbs, and Aromatic and Medicinal Plants of the Temperate Zone. Archon Books, 770 pp., Hamden, CT.

$$
\text { الملخص العربي }
$$

\section{تأثير التسميد المعدني والعضوي والحيوي على محصول وجودة البقدونس}

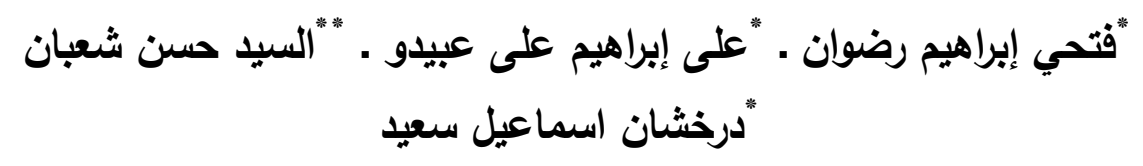

* قسم الإنتاج النباتي ـ كلية الزراعة سابا باشا ـ جامعة الإسكندرية ـ مصر

* قسم بحوث النباتات الطبية والعطرية - مركز البحوث الزراعية - شعبة إنتاج وتكنولوجيا النباتات

$$
\text { الطبية والعطرية. }
$$

أجريت تجربتان حقليتان خلال موسمي الزراعة 2013/2012 ، 2014/2013 لدراسة تأثير التسميد الكيماوي ومعدلات السماد العضوي والحيوي على صفات النمو الخضري الحيوي الكيماوي ومكونات محتوي الزيت لنبات البقدونس وقد استخدم في هذه التجارب التصميم الإحصائي القطاعات العشوائية الكاملة لنلاث مكررات. ويمكن تلخيص أهم النتائج فيما بليذ

- أدى استخدام معاملات التسميد لتأثنرات معنوية مختلفة على أعلى متوسط لقيم جميع الصفات المدروسة أيضاً. - أدى إضافة المعاملة (7) 2 طن سماد عضوي/فدان + 50\% نتروجين فوسفور بوتاسيوم + سيريالين +

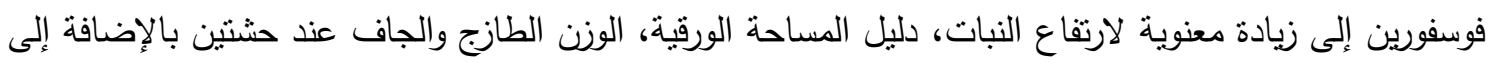

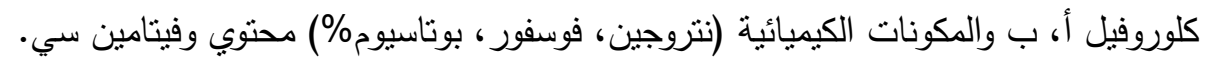

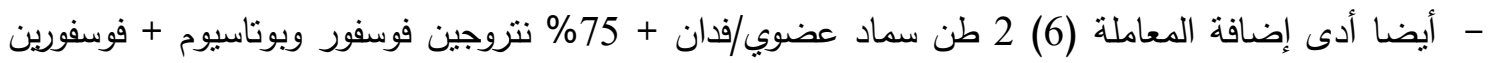
إلى ارتفاع مكونات محتوى الزيت (النسبة المئوية للمكونات) في الموسم الأول 2013/2012. نوصي باستخدام 2 طن سماد عضوي /ف + 50 + 50+ 25ك/ف من من سلفات النشادر وسوبر فوسفات الكالسيوم وسلفات البوتاسيوم على التوالي + سيريالين + فوسفورين للحصول على أفضل نمو وجودة نبات البقدونس. 
